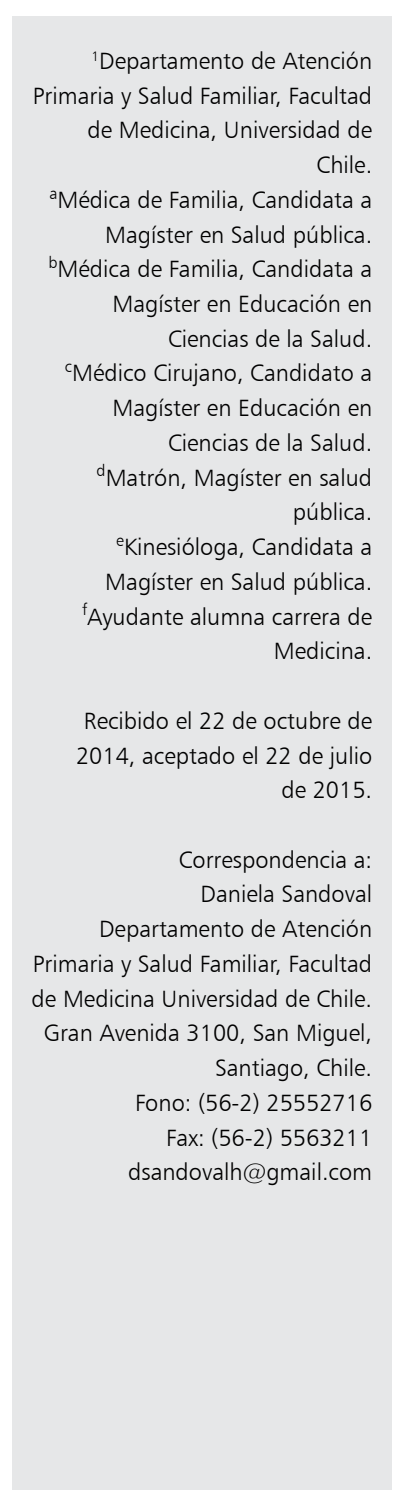

\section{Actividad laboral de médicos egresados de la Universidad de Chile generaciones 2007 a 2010: análisis según sede y año de egreso}

\author{
PATRICIA VARGAS ${ }^{1, \mathrm{a}}$, NANCY LÓPEZ ${ }^{1, \mathrm{~b}}$, LEONARDO URRUTIA $^{1, \mathrm{c}}$, \\ JHONNY ACEVEDO ${ }^{1, \mathrm{~d}}$, DANIELA SANDOVAL ${ }^{1, \mathrm{e}}$, BELÉN CORTÉS $^{1, \mathrm{f}}$
}

\section{Work activity of medical graduates at the University of Chile. Analysis per campus and graduation year}

\begin{abstract}
Background: The University promotes practices and values that influence their students in the pursuit of their occupational interests. Aim: To determine working activity features of medical graduates from the University of Chile and their relationship with undergraduate characteristics. Material and Methods: Medical graduates of the University of Chile were invited to complete a survey using a virtual server. The survey collected demographic, socioeconomic, work and guild characteristics. Undergraduate data, as campus and graduation years were obtained. Results: The survey was completed by 333 physicians (167 men) aged $29 \pm 2$ years, graduated from 2007 to 2010 . Ninety four percent had a paid work, $59 \%$ were employed in public hospitals and $28 \%$ in primary care health centers. The predominant type of work activity was performed in the public health service (55\%), while $17 \%$ worked in private health services. Activity in public health services or primary care were significantly associated with the pursuit of undergraduate clinical activities in specific campuses of the University. Conclusions: The employment rate of medical graduates was high. Differences observed by graduation campus and type of work may be due to the modeling that students receive from teachers.
\end{abstract}

(Rev Med Chile 1286-1294)

Key words: Employment; Private practice; Public health.

\section{L} a universidad, por ser una institución educativa, fomenta ciertas prácticas y valores que se imprimen en sus estudiantes. Estos valores influyen en la visión del egresado al momento de buscar sus intereses laborales y desde ahí su posición en la sociedad.

La Facultad de Medicina de la Universidad de Chile, fundada en 1842, ha sido pionera en la formación de médicos de gran capacidad, con sólida formación ética, espíritu de servicio y compromiso con el bienestar de la comunidad según lo declara su misión institucional ${ }^{1}$. En su compromiso por alcanzar calidad y excelencia se interesa por la empleabilidad y por la condición laboral de sus egresados por cuanto son el reflejo de una necesidad social en salud, en los ámbitos de la atención pública y privada ${ }^{2,3}$ que permite la adecuación de los perfiles de egreso de los médicos en formación $n^{4,5}$.

La ocupación del médico egresado es mencionado como uno de los criterios de "Los Estándares Globales en Educación Médica” de la Federación 
Mundial para la Educación Médica ${ }^{6}$, que considera entre sus propósitos la preparación de médicos que respondan a las necesidades y expectativas de la sociedad. Muñoz y cols ${ }^{4}$ agregan que las carreras de medicina además de cumplir con la sociedad, proporcionando información confiable en cuanto a la calidad de sus programas de estudio, también deben responder a sus estudiantes respecto de la empleabilidad y ocupación a la que razonablemente podrán aspirar a su egreso. Sin embargo, esta información a menudo es difícil de hallar.

Hasta donde ha sido posible indagar en Chile no se han encontrado estudios publicados que muestren las características de la ocupación de los médicos luego de egresar de su programa de formación, especialmente dentro de los primeros 5 años de su ejercicio profesional. Y no existen datos previos acerca de los egresados de la Facultad de Medicina de nuestra universidad.

En consecuencia, el presente estudio tiene como objetivo determinar las características de la actividad laboral de médicos egresados de la Universidad de Chile entre los años 2007 y 2010, para conocer el estado personal del egresado y aspectos de su ejercicio profesional, en particular si este es ejercido en el servicio público o privado de la atención de salud y evaluar si existen relación entre estas condiciones y características propias del pregrado como la sede y el año de egreso.

\section{Material y Método}

\section{Diseño y población de estudio}

El estudio observacional de corte transversal, en una muestra de 333 médicos egresados de la Facultad de Medicina de la Universidad de Chile entre los años 2007 y 2010. La muestra consideró a todos los sujetos que aceptaron participar tras una campaña de difusión del estudio en la cual se invitó al universo de 793 médicos egresados entre dichos años.

Los datos de contacto (teléfonos, e-mail y redes sociales) de los 793 médicos, se obtuvieron a través del registro docente recopilado durante el paso de los estudiantes por la asignatura "Internado Urbano de Atención Primaria en Salud”, impartida por el Departamento de Atención Primaria y Salud Familiar de la Facultad de Medicina. Dicha asignatura es de carácter obligatorio para todos los estudiantes de la carrera.
La campaña de difusión e invitación al estudio incluyó el envió de invitaciones por e-mail, por contacto a través de redes sociales creadas por el estamento estudiantil como redes de ex alumnos (Facebook), y por contacto telefónico.

Se pidió a los participantes que contestaran una encuesta semi-estructurada usando un servidor virtual. La encuesta fue diseñada por el equipo investigador, la que se cotejó con otras encuestas de estudios similares para el diseño definitivo ${ }^{7,8,9}$. La encuesta fue contestada de forma personal, voluntaria y enviada a través de un servicio on-line que resguardó la privacidad y confiabilidad de los datos de los encuestados. La encuesta estuvo disponible para ser respondida durante dos meses el segundo semestre del año 2013.

\section{Mediciones}

La encuesta recogió antecedentes de tres tipos:

1) Antecedentes personales: que incluía la identificación del encuestado, edad, sexo, estado civil, año y sede de egreso universitario.

2) Ámbito profesional actual, que consideró las siguientes preguntas: ¿ejerces actualmente como médico?, ¿en qué área de la atención de salud trabajas?, ¿cuántas horas semanales comprende tu jornada laboral?, ¿cuántas horas trabajas en el sistema público de salud?, ¿cuántas horas trabajas en el sistema privado de salud?, ¿específicamente en qué tipo de establecimiento trabajas?, ¿has realizado alguna capacitación de posgrado?.

3) Aspectos económicos y gremiales: que consideró las preguntas ¿en qué tramo de ingresos mensuales se ubica tu renta actual?, ¿estás inscrito en el Colegio Médico?

Los antecedentes personales fueron recogidos a través de respuestas abiertas. Todas las demás preguntas fueron cerradas, con 2 a 5 alternativas de respuestas categóricas según tipo de pregunta. Sólo la pregunta ¿Específicamente en qué establecimiento trabajas? consideró alternativas múltiples y no excluyentes.

La variable "área de atención de salud" en la cual se desempeña el egresado, se construyó a partir de tres preguntas de la encuesta, su construcción final consideró 4 categorías: "Público" y "Privado" según la mayor cantidad de horas de dedicación al sector de atención en salud. "Mixta" en aquellos casos en que el egresado dedicaba la 
misma cantidad de horas a cada sector, y "Otras áreas" que consideró a quienes realizaban prestaciones profesionales de otras índoles como docencia.

La actividad laboral ejercida en centros de Atención Primaria de Salud (APS), consideró a quienes trabajaban en establecimientos de salud de nivel primario como Centros de Salud Familiar (CESFAM), Centros de Salud, Postas Rurales, Servicios de Atención Primaria de Urgencia (SAPU) y Centros Comunitarios de Salud Mental (COSAM).

\section{Análisis estadístico}

Se utilizó el programa estadístico SPSS versión 15.0 en español para la exploración y análisis de los datos. La valoración de los resultados se expresó a través de frecuencias absolutas y relativas para las variables categóricas y de media y desviación estándar para las variables continuas. Para determinar las diferencias de las variables evaluadas según año de egreso o según sede de egreso, se utilizó prueba de $\chi^{2}$ para las variables categóricas y t-Student para las variables continuas. Para determinar la asociación entre la actividad laboral ejercida en atención pública y sede de egreso se realizó análisis de regresión logística binaria, obteniéndose $O d d s$ Ratio (OR) con intervalos de confianza de 95\% (IC 95\%). La variable dependiente se categorizó como 0 cuando la actividad laboral no era ejercida en atención pública de salud, y como 1 cuando si era ejercida en ese nivel de atención. La variable sede de egreso se categorizó como 1 para cada sede de egreso y como 0 para la agrupación de sedes restantes. El mismo análisis se realizó para determinar la asociación entre la actividad laboral ejercida en APS y la sede de egreso.

\section{Resultados}

Del total de 793 médicos egresados entre los años 2007 a 2010, que fueron invitados a participar, sólo 333 médicos (42\%) contestaron íntegramente la encuesta dentro del plazo establecido y fueron incluidos en el estudio. Un 50,2\% ( $n=167)$ de los encuestados fueron hombres. La edad promedio fue de $28,8 \pm 1,8$ años, sin diferencias significativas entre hombres y mujeres $(p=0,98)$.

La Tabla 1 muestra la distribución de los médicos según sede y año de egreso, en ambas no se observaron diferencias significativas entre la distribución de la muestra y el universo.

El estado civil de los médicos egresados más frecuente fue el estado de soltero (55\%). Una amplia mayoría, $94,3 \%(\mathrm{n}=314)$ se encontraba ejerciendo su profesión al momento de contestar la encuesta. El 5,7\% $(n=19)$ restante declaró no ejercer una actividad laboral remunerada al encontrarse cursando programa de especialización médica $(n=6)$, o programas de postgrado nacional o internacional de dedicación exclusiva $(n=9)$ o desarrollando actividades docentes y de investigación $(\mathrm{n}=4)$.

En cuanto a la actividad académica de postgrado, $51 \%$ ha realizado o se encontraba realizando una especialidad médica y $26 \%$ había realizado un diplomado en alguna área de interés. En este ítem se encontraron diferencias por sexo en donde las mujeres superan a los hombres en la realización de especialidad médica $(57,9 \%$ vs $44,3 \%$ respectivamente; $\mathrm{p}=0,01)$ (Tabla 2).

Tabla 1. Comparación de la distribución de médicos encuestados (muestra) y la distribución del total de médicos egresados de la Universidad de Chile entre los años 2007 y 2010 (universo), según sede y año de egreso

\begin{tabular}{|c|c|c|c|c|c|c|c|}
\hline \multicolumn{5}{|c|}{ Sede de Egreso } & \multicolumn{3}{|c|}{ Año de egreso } \\
\hline & $\begin{array}{c}\text { Muestra } \\
\% \quad \text { (n) }\end{array}$ & $\begin{array}{c}\text { Universo } \\
\% \quad \text { (n) }\end{array}$ & p-value & & $\begin{array}{c}\text { Muestra } \\
\% \quad \text { (n) }\end{array}$ & $\begin{array}{c}\text { Universo } \\
\% \quad \text { (n) }\end{array}$ & p-value \\
\hline Norte & $32,4(108)$ & $31,4(249)$ & 0,73 & & & & \\
\hline Sur & $12,3 \quad(41)$ & $12,7(100)$ & 0,84 & 2007 & $20,4 \quad(68)$ & $25,2(200)$ & 0,08 \\
\hline Centro & $16,2 \quad(54)$ & $16,1(128)$ & 0,97 & 2008 & $23,1 \quad(77)$ & $23,3(185)$ & 0,94 \\
\hline Oriente & $19,5 \quad(65)$ & $21,4(170)$ & 0,46 & 2009 & $26,7 \quad(89)$ & $23,1(183)$ & 0,19 \\
\hline \multirow[t]{2}{*}{ Occidente } & $19,5 \quad(65)$ & $18,4(146)$ & 0,66 & 2010 & $29,7 \quad(99)$ & $28,4(225)$ & 0,65 \\
\hline & $100(333)$ & $100(793)$ & & & $100(333)$ & $100(793)$ & \\
\hline
\end{tabular}


Tabla 2. Características sociodemográficas, laborales y económicas de los médicos egresados de la Universidad de Chile entre los años 2007 a 2010. Comparación según sexo

\begin{tabular}{|c|c|c|c|c|}
\hline Características & $\begin{array}{c}\text { Total } \\
(n=333)\end{array}$ & $\begin{array}{l}\text { Hombre } \\
(n=167)\end{array}$ & $\begin{array}{l}\text { Mujeres } \\
(n=166)\end{array}$ & p-value \\
\hline \multicolumn{5}{|l|}{ Sociodemográficas } \\
\hline Edad promedio (años) & $28,8 \pm 1,8$ & $28,7 \pm 1,8$ & $28,7 \pm 1,8$ & 0,98 \\
\hline \multicolumn{5}{|l|}{ Estado civil (\%) } \\
\hline Soltero & 55,0 & 55,1 & 54,8 & 0,96 \\
\hline Casado/conviviente & 43,8 & 43,7 & 44,0 & 0,96 \\
\hline Separado & 1,2 & 1,2 & 1,2 & 0,62 \\
\hline \multicolumn{5}{|l|}{ Laborales (\%) } \\
\hline ¿Ejerces actualmente como médico? & 94,3 & 94,0 & 94,6 & 0,82 \\
\hline \multicolumn{5}{|l|}{ ¿Cuándo comenzaste a trabajar? } \\
\hline Antes de egresar & 8,4 & 9,0 & 7,8 & 0,70 \\
\hline Inmediatamente después de egresar & 55,0 & 53,9 & 56,0 & 0,69 \\
\hline Un par de meses después de egresar & 33,3 & 34,7 & 31,9 & 0,59 \\
\hline Seis meses después de egresar & 3,3 & 2,4 & 4,2 & 0,35 \\
\hline \multicolumn{5}{|c|}{ ¿Cuánto tiempo demoraste en encontrar trabajo? } \\
\hline Menos de 1 mes & 89,8 & 90,4 & 89,2 & 0,70 \\
\hline Entre 1 y 6 meses & 9,9 & 9,6 & 10,2 & 0,84 \\
\hline Más de 6 meses & 0,3 & - & 0,6 & - \\
\hline \multicolumn{5}{|c|}{ ¿Cuántas horas semanales comprende tu jornada laboral? } \\
\hline Menos de $22 \mathrm{~h}$ semanales & 5,4 & 4,2 & 6,6 & 0,32 \\
\hline De 22 a 33 h semanales & 7,5 & 4,8 & 10,2 & 0,06 \\
\hline De 33 a 44 h semanales & 26,4 & 22,2 & 30,7 & 0,07 \\
\hline Más de $44 \mathrm{~h}$ semanales & 60,7 & 68,9 & 52,4 & 0,002 \\
\hline \multicolumn{5}{|c|}{ Específicamente ¿en qué establecimientos trabajas?* } \\
\hline Hospital público & 58,9 & 57,5 & 60,2 & 0,60 \\
\hline Clínica privada & 19,8 & 22,2 & 17,5 & 0,28 \\
\hline Establecimiento de Atención Primaria & 28,2 & 26,3 & 30,1 & 0,44 \\
\hline Centro médico privado & 16,8 & 16,8 & 16,9 & 0,98 \\
\hline Institución académica & 14,7 & 18,0 & 11,4 & 0,09 \\
\hline \multicolumn{5}{|c|}{$\begin{array}{l}\text { Académicas } \\
\text { ¿Haz realizado alguna capacitación de posgrado? }\end{array}$} \\
\hline Especialidad Médica & 51,0 & 44,3 & 57,9 & 0,01 \\
\hline Diplomado & 26,4 & 31,1 & 21,7 & 0,05 \\
\hline Magíster & 8,7 & 8,4 & 9,0 & 0,83 \\
\hline Doctorado & 0,9 & 1,8 & -- & \\
\hline Otros (cursos de actualización) & 3,3 & 3,6 & 3,0 & 0,76 \\
\hline Ninguno & 9,6 & 10,8 & 8,4 & 0,47 \\
\hline \multicolumn{5}{|l|}{ Económicas } \\
\hline \multicolumn{5}{|c|}{ ¿En qué tramo de ingresos mensuales te ubicas? } \\
\hline Menos de 500.000 pesos & 9,9 & 8,4 & 11,4 & 0,35 \\
\hline Entre 500.000 y 750.000 pesos & 6,9 & 8,4 & 5,4 & 0,29 \\
\hline Entre 750.000 y 1 millón & 8,7 & 6,0 & 11,4 & 0,08 \\
\hline Entre 1 millón y 2 millones & 47,4 & 42,5 & 52,4 & 0,07 \\
\hline Más de 2 millones & 22,5 & 28,7 & 16,3 & 0,006 \\
\hline Respuestas omitidas & 4,5 & 6,0 & 3,0 & 0,19 \\
\hline \multicolumn{5}{|l|}{ Gremiales } \\
\hline \multicolumn{5}{|l|}{ ¿Estas inscrito en el colegio médico? } \\
\hline Sí & 55,9 & 53,3 & 58,4 & 0,34 \\
\hline No & 44,1 & 46,7 & 41,6 & 0,34 \\
\hline
\end{tabular}

*Categorías no excluyentes. 
El tipo de jornada laboral predominante fue el de más de $44 \mathrm{~h}$ semanales $(60,7 \%)$, en la que se observó diferencias según sexo $(68,9 \%$ en hombres vs $52,4 \%$ en mujeres; $\mathrm{p}=0,002)$. Respecto al comienzo del trabajo 63,4\% declaró haber comenzado inmediatamente al momento del egreso o antes de egresar (Tabla 2).

De acuerdo al tipo de establecimiento en que se desempeñaban los médicos egresados 58,9\% ejercía su profesión en hospitales públicos, 28,2\% en centros de APS y 19,8\% en clínicas privadas (Tabla 2). No se encontraron diferencias según sexo.

Respecto al tramo de ingresos mensuales percibidos más de $50 \%$ de los encuestados percibe una renta mayor a un millón de pesos mensuales. Se observó diferencias respecto al sexo en el tramo de ingresos mayor a los 2 millones de pesos mensuales en donde los hombres poseen un proporción mayor $(28,7 \%$ vs $16,3 \% ; \mathrm{p}=0,006)$. En análisis diferenciado por sede de egreso no se hallaron diferencias significativas en el inicio del trabajo, ni en la cantidad de horas en la jornada laboral, ni en el tramo de ingresos percibidos mensualmente (Tabla 3).

Respecto de la variable área o sector de la atención de salud, $54,7 \%$ se desempeñan en el sector público, $16,5 \%$ en el sector privado, $18,3 \%$ en ambos sectores y 10,5\% en otros (docencia). En análisis según sede de egreso se observó una proporción menor de médicos trabajando en el sector de atención pública de salud entre quienes egresan de la sede campus norte en comparación con quienes lo hacen desde otras sedes (Figura 1). Este hecho se comprueba en análisis de regresión logística donde los médicos egresados desde el campus norte se relacionan de manera inversa con el ejercicio laboral en la atención pública de salud (OR 0,49; IC 95\% 0,31-0,77) (Tabla 4).

Tabla 3. Características generales sobre ámbito profesional y económico de los médicos egresados de la Universidad de Chile según sede de egreso

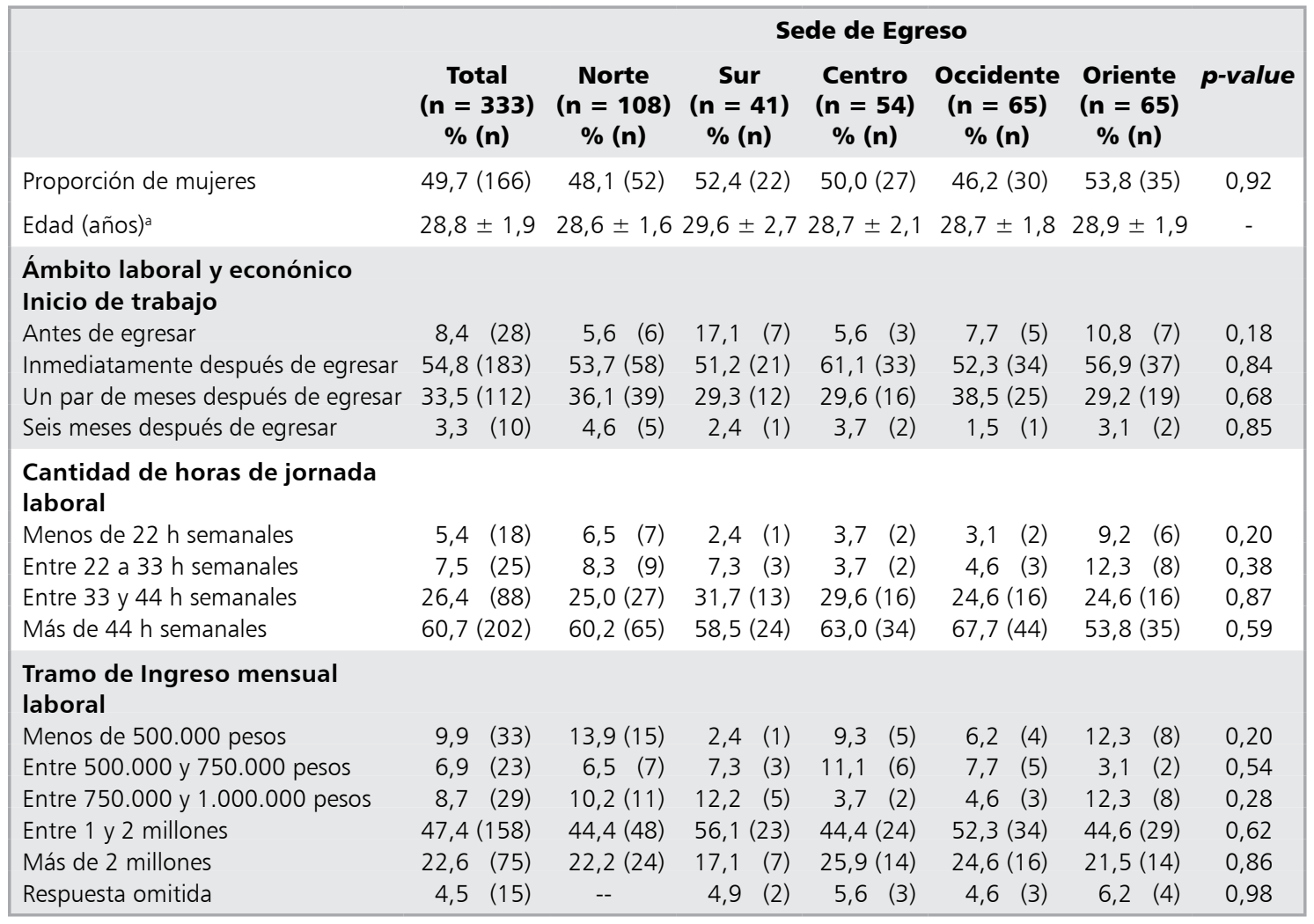

aalores expresados en promedios \pm desviación estándar. 


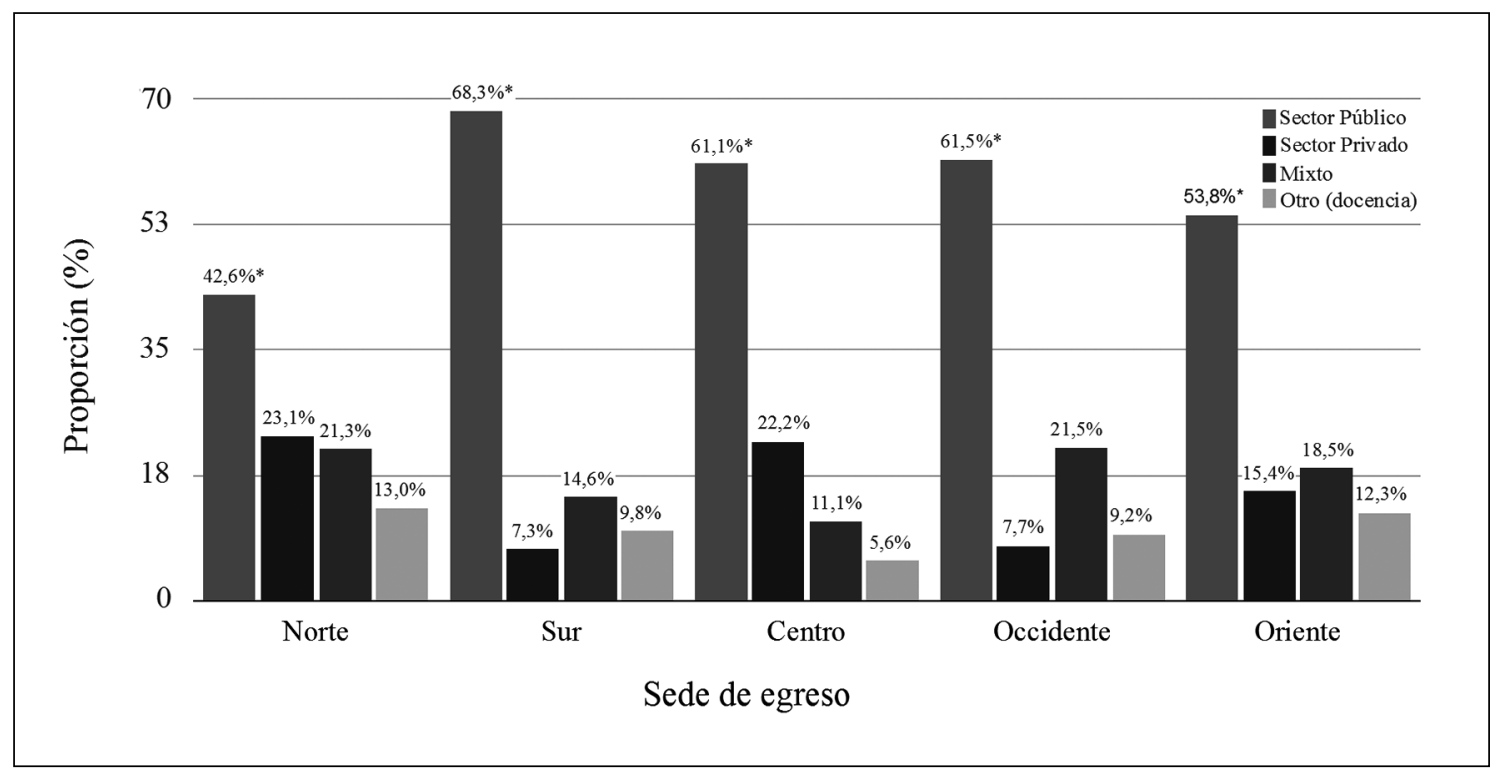

Figura 1. Distribución del área de atención de salud en la cual trabajan los médicos egresados de la Universidad de Chile entre los años 2007 a 2010, según sede de egreso (*p <0,05).

Tabla 4. Análisis de Regresión Logística (OR con IC 95\%) para la asociación entre sede de egreso y la actividad laboral ejercida en atención pública y la ejercida en centros de Atención Primaria de Salud (APS) de los médicos egresados de la Universidad de Chile entre los años 2007 a 2010

\begin{tabular}{|lccccc|}
\hline & Sede Norte & Sede Sur & $\begin{array}{c}\text { OR (IC 95\%) } \\
\text { Sede Centro }\end{array}$ & Sede Oriente & Sede Occidente \\
\hline Actividad laboral en Atención & 0,49 & 1,93 & 1,37 & 0,96 & 1,42 \\
Pública de Salud & $(0,31-0,77)$ & $(0,96-3,87)$ & $(0,76-2,49)$ & $(0,56-1,65)$ & $(0,85-2,47)$ \\
Actividad Laboral en APS & 0,66 & 1,81 & 1,75 & 0,42 & 1,44 \\
& $(0,39-1,09)$ & $(0,93-3,53)$ & $(0,96-3,18)$ & $(0,21-0,82)$ & $(0,82-2,53)$ \\
\hline
\end{tabular}

La Figura 2 muestra la actividad laboral ejercida en APS, según sede de egreso. Las sedes de egreso norte y oriente presentaron menor proporción de médicos trabajando en centros de APS. Sólo la sede campus oriente mostró asociación significativa en análisis de regresión logística que relacionó la sede de egreso con el desempeño profesional en centros de APS (OR 0,42; IC 95\% 0,21-0,82) (Tabla 4). Finalmente, la Figura 3 muestra la distribución de la actividad laboral ejercida en APS según año de egreso, en que se observó una tendencia de mayor proporción de médicos dedicados a esa área de la atención de salud en las generaciones más jóvenes.

\section{Discusión}

Uno de los aspectos más relevantes encontrados en nuestro estudio fue la alta empleabilidad de los médicos egresados de nuestra facultad (94,3\%), cifra similar a lo informado por el Ministerio de Educación para la carrera de medicina que menciona una tasa de empleabilidad de $94,0 \%$ al primer año y $95,1 \%$ al segundo año posterior a la titulación ${ }^{10}$. Otro dato de interés es que nuestros egresados acceden rápidamente al empleo, más de $60 \%$ encuentra trabajo inmediatamente después de egresar o incluso antes.

Respecto de los establecimientos de atención 

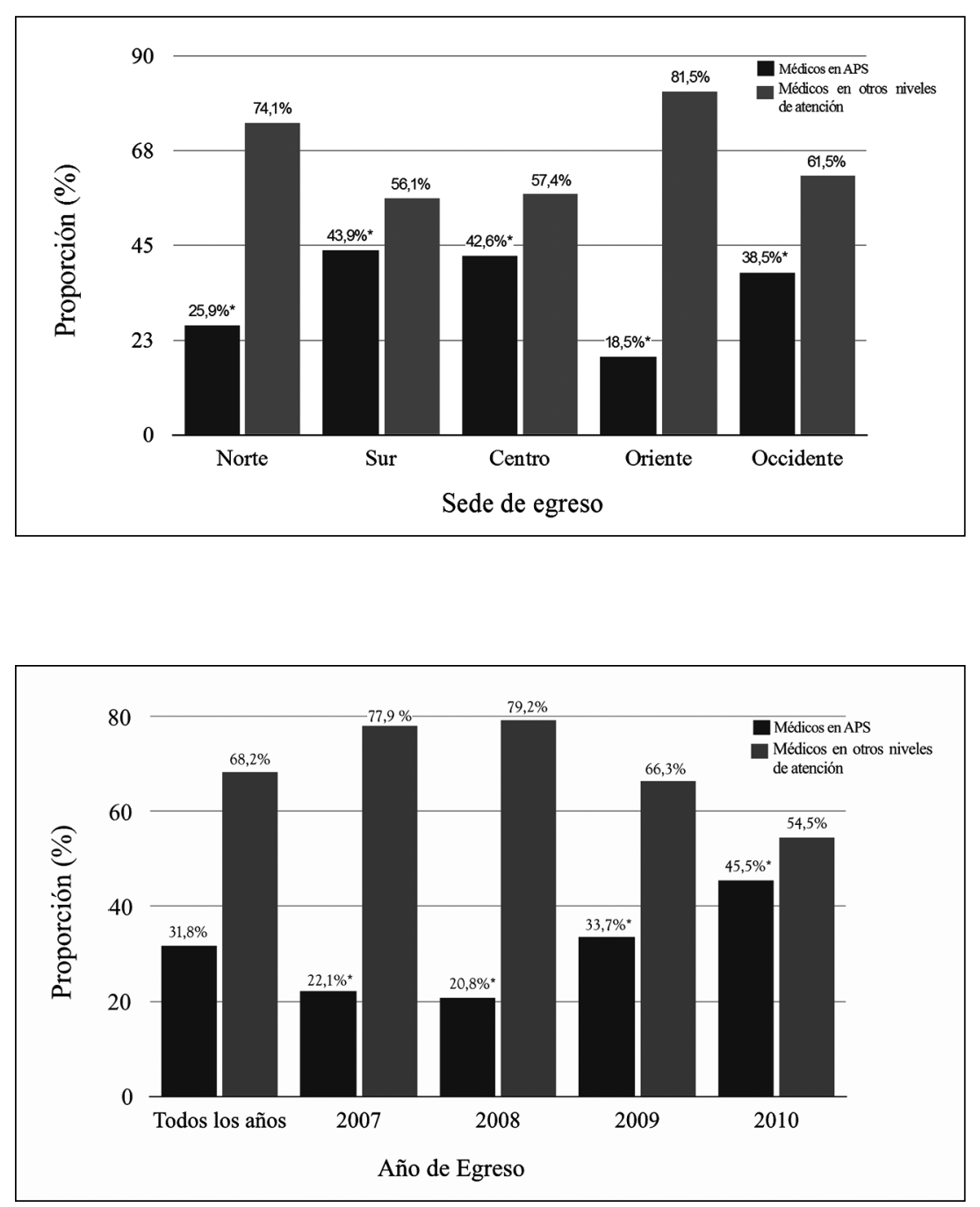

Figura 2. Proporción de médicos egresados de la Universidad de Chile entre los años 2007 a 2010 que trabajan en centros de Atención Primaria de Salud (APS), comparación según sede de egreso ( $\left.{ }^{*} p<0,01\right)$.
Figura 3. Proporción de médicos egresados de la Universidad de Chile entre los años 2007 a 2010 que trabajan en centros de Atención Primaria de Salud (APS), comparación según año de egreso ( $\left.{ }^{*} p<0,001\right)$. en salud donde los médicos egresados ejercen su profesión, nuestro estudio mostró una mayor proporción de médicos en el ámbito hospitalario en comparación con el ejercicio profesional en centros de APS (58,9\% vs $28,2 \%)$. La baja proporción de médicos en este nivel de atención, ha sido mencionada por otros autores que desde el año 2005 hacían notar un déficit preocupante de horas médicas en este nivel de atención ${ }^{11}$. Un estudio más reciente mostró que el déficit de horas médicas aún persiste, con remuneraciones insuficientes y con un status de menoscabo respecto a otros niveles de atención en salud como la atención hospitalaria ${ }^{12}$. Entre los posibles factores asociados a este hecho estudios señalan la bajas remuneraciones, la falta de carrera profesional y la baja preparación en competencias profesionales para la APS en comparación con la fuerte formación "hospitalocéntrica" recibida en los programas de formación ${ }^{12,13}$. Al respecto en Latinoamérica, los modelos de formación de médicos de las distintas escuelas de medicina se basaron durante décadas en los lineamientos de Abraham Flexner que proponía el rol del médico en torno al tratamiento de la enfermedad y la especialización de la práctica médica, por tanto, la formación de médicos tenía sentido en el ambiente hospitalario. Posteriormente la doctora María Rosa Borrell mencionó 
la necesidad de cambio en los programas de formación actuales a otros basados en competencias incorporando una visión integral de la atención del paciente, en consecuencia los escenarios formativos del médico debían ampliarse hacia la APS $^{14}$. Siguiendo esta línea de pensamiento, desde el año 2011 nuestra Facultad ha incorporado en su programa de formación un currículum innovado con una visión que incorpora la formación en medicina en el nivel primario de atención como eje central, por tanto a futuro sería interesante evaluar si la proporción de médicos en centros de APS de las generaciones con currículum innovado varia respecto a las generaciones estudiadas en esta investigación.

Respecto al análisis según sede de egreso, la sede oriente se asoció de manera inversa con la actividad laboral ejercida en la APS. Tradicionalmente esta sede posee una larga trayectoria de trabajo en Hospital del Salvador, Instituto Nacional del Tórax e Instituto de Neurocirugía destacando además la preparación de la enseñanza de Pediatría en Hospital Luis Calvo Mackenna, que acentúa la relación docente asistencial de esta sede con los recintos hospitalarios por lo que la impronta formativa de sus estudiantes está fuertemente influenciada por este nivel de atención. Si bien el cambio curricular anteriormente descrito es transversal a todos los campus clínicos de la Facultad de Medicina de nuestra Universidad, sería interesante conocer a futuro si las generaciones con currículum innovado de esta sede cambia la tendencia observada.

Otro hallazgo interesante es que las generaciones más jóvenes 2009-2010 poseen una proporción mayor de médicos desempeñándose en APS que las generaciones anteriores (Figura 3). Hipótesis que pudieran dar cuenta de este hallazgo van entorno a que paulatinamente las políticas gubernamentales han fortalecido la APS dando apertura a un modelo de atención biopsicosocial que pudiera ser más atractivo para médicos de egreso reciente, quienes prefieren ingresar al mercado laboral en esta área a la espera de incorporarse en programas de especialización médica. Dicha hipótesis sería interesante de corroborar en estudios posteriores.

Respecto al análisis de la actividad laboral ejercida por estas generaciones, se observó una proporción mayor del ejercicio profesional en el ámbito público (54,7\%). Esta cifra difiere con $44,0 \%$ de contratos médicos en el sector público informado por la subsecretaria de redes asistenciales el año 2008 para el país ${ }^{16}$.

Las sedes de egreso norte y oriente mostraron menor proporción de médicos trabajando en el sector público en comparación con las demás sedes, sin embargo, sólo la sede norte mostró asociación inversa y significativa en análisis de regresión logística con el ejercicio laboral en el servicio público. La sede campus norte es la más antigua, y es además campus de formación de otros profesionales de salud a diferencia de las demás sedes de egreso, sin embargo, las posibles explicaciones de este hallazgo escapan al análisis que pueden realizarse con los datos recopilados en este estudio.

En conclusión, este estudio proporciona una descripción sobre las características de la actividad laboral de los médicos egresados de la Universidad de Chile de interés académico, por cuanto la impronta de esta institución es eminentemente pública y social. Se visualiza un alto índice de empleo con tendencia laboral al ejercicio profesional principalmente en el servicio público de salud con una proporción importante de médicos incluidos en hospitales en comparación con los médicos de APS.

\section{Referencias}

1. Misión de la Facultad de Medicina de la Universidad de Chile. Facultad de Medicina. Universidad de Chile. 2015. Disponible en: http://www.med.uchile.cl.

2. Rosselot E, Mateluna E, Estévez A, Radrigán ME. Acreditación de título de profesionales e Instituciones formadoras: el caso de Chile. Rev Med Chile 1998; 126: 715-24.

3. Román O, Señoret M. Estado actual de las especialidades médicas en Chile; realidad en el sistema público no municipalizado. Rev Med Chile 2008; 136: 99-106.

4. Muñoz M, Moreno R, Hanne C, Pantoja M, Altamirano P. Ocupación de los egresados de la carrera de medicina: Relevancia del diseño curricular. Rev Educ Cienc Salud 2009; 6 (2): 75-8.

5. Organización Mundial de la Salud y Federación Mundial para la Educación Médica. Ginebra y Copenhague, 2005. Guía de la OMS/WFME para la acreditación de la formación médica de grado. Edu Med 2006; 9 (3): 103-10.

6. Estándares Globales en Educación Médica de la WFME (Federación Mundial para la Educación Médica). Edu. Med 2004 v.7 supl.2. Barcelona jul-sep. 
7. Millán T, Ercolano M, Pérez M, Fuentes C. Autoevaluación de habilidades clínicas básicas en médicos recién egresados de la Facultad de Medicina, Universidad de Chile. Rev Med Chile 200 ; 135: 1479-86. Disponible en: http://www.scielo.cl/scielo.php?script=sci_arttext\&pi$\mathrm{d}=$ S0034-98872007001100017.

8. Palés J, Valles A, Cardellach F, Gomar C, Estrach M, Cost $\mathrm{J}$, et al. Habilidades y procedimientos clínicos básicos a adquirir por los estudiantes de la Facultad de Medicina de la Universidad de Barcelona. Edite Med 2001; 4: 72-81.

9. Torres S, Camacho A, López J, Sánchez P, Pérez F. El proceso docente de adquisición de habilidades clínicas en la Universidad de Córdova: Una autoevaluación de los alumnos. Educ Med 2003; 6: 117-22.

10. Futuro Laboral. Ministerio de Educación. Gobierno de Chile. Servicio de información de Educación Superior (SIES). 2015. Disponible en www.mifuturo.cl.

11. Castro JL. El déficit de los médicos en la atención primaria urbana. Cuad Méd Soc (Chile) 2005: 45 (4): 241-2.
12. Montero J, Tellez A, Herrera C. La reforma sanitaria chilena y la Atención Primaria en Salud. Algunos aspectos críticos. Temas de la Agenda Pública 2010; 5 (38): Santiago: Pontificia Universidad Católica de Chile.

13. Román $\mathrm{O}$, Pineda $\mathrm{S}$, Señoret M. Pérfil y número de médicos generales que requiere el país. Rev Med Chile 2007, 135: 1209-15.

14. Borrell Bentz MR. La educación médica de posgrado en Argentina: el desafío de una nueva práctica educativa. $1^{\text {a }}$ ed. Buenos Aires: Organización Panamericana de la Salud-OPS, 2005.

15. Universidad de Chile. Facultad de Medicina. Descripción de los campos formativos de la Facultad de Medicina: Campus Oriente. Disponible en: http://www.med. uchile.cl/campus-oriente.html.

16. Guillou M. Estudio de brechas de demanda y oferta de médicos especialistas en Chile. Informe final. Banco mundial. Gobierno de Chile-Ministerio de Salud. Enero 2010. Disponible en: http://ciperchile.cl/wp-content/ uploads/Estudio_subse_redes_banco_mundial.pdf 